\title{
Analysis of Body Sensor Network Using Human Body as the Channel
}

\author{
Jerald Yoo, Namjun Cho, Hoi-Jun Yoo \\ Korea Advanced Institute of Science and Technology (KAIST) \\ 1233 Electrical Engineering, KAIST \\ 373-1, Guseong, Yuseong, Daejeon, South Korea \\ 82-42-869-5468 \\ \{jerald, buzm\}@eeinfo.kaist.ac.kr, hjyoo@ee.kaist.ac.kr
}

\begin{abstract}
A body sensor network using human body as a communication medium is analyzed and designed to achieve both power- and energy-efficiency. An analysis of the body channel network on frequency, distance, transmitting power and received power is performed. The analysis reveals the star topology consumes less energy than the ad-hoc topology for body channel network. Based on the analysis results, the packet structure for body channel network, with variable payload size that minimizes energy consumption, is designed.
\end{abstract}

\section{Categories and Subject Descriptors}

A.1 [General Literature]: Introductory and Survey; C.2.0 [Computer Systems Organization]: Computer-communication Networks - General

\section{General Terms}

Measurement, Design, Experimentation

\section{Keywords}

Body sensor network, body channel communication, body channel network, star topology

\section{INTRODUCTION}

Recently, there have been active researches on the body channel communication (BCC), using human body as a communication medium [2], [7]-[13]. Since BCC operates as near-field coupling, the transmitting signal is limited to near body area and do not interfere with external RF devices. In addition, since the communication frequency can be lowered without increasing the antenna size, the transmitter power can be reduced much more compared to the Bluetooth or ZigBee radios [13]. These works showed good insights in understanding BCC, and opened the door for prospective applications of BCC such as healthcare systems, biomedical applications and multimedia systems [3], [8]-[12].

Permission to make digital or hard copies of all or part of this work for personal or classroom use is granted without fee provided that copies are not made or distributed for profit or commercial advantage and that copies bear this notice and the full citation on the first page. To copy otherwise, to republish, to post on servers or to redistribute to lists, requires prior specific permission and/or a fee.

BodyNets 2008, March 13-15 Tempe, Arizona, USA

Copyright $\odot 2008$ ICST 978-963-9799-17-2

DOI 10.4108/ICST.BODYNETS2008.2928
Unfortunately, no study has been reported yet how to form an energy-efficient network with BCC. To implement body sensor network applications, energy efficiency is the key issue due to the battery limitations of the wearable/implantable sensor nodes.

Figure 1 shows the concept of using BCC to form a body channel network (BCN). A base station controls the network and collects bio signal data from all sensor nodes. The test system is shown (a base station) with temperature, blood volume pulse (BVP) and other sensor nodes worn on a dummy.

In this paper, a $\mathrm{BCN}$ for healthcare system is analyzed in terms of frequency, distance, transmitting power and received power. Furthermore, an energy-efficient network protocol using BCC is designed and analyzed. In section 2, the body channel characteristics and the path loss equation are shown. Section 3 analyzes the BCN in distance, frequency and transmitting/received power. Topology comparison between simple star and simple ad-hoc is also described. In section 4 , an energy-efficient packet structure for the BCN is designed and analyzed. Finally, section 5 concludes the paper.

\section{BODY CHANNEL CHARACTERISTICS}

\subsection{The Body Channel}

Zimmerman's model [13] describes the near-field coupling around the human body as a current loop formed by the transmitter electrode, the body channel, the receiver electrode and the capacitive coupled return path. In his model, the human body was assumed as a perfect conductor, and the body was approximated into a single node. This mechanism fits well for low communication frequencies where the impedance of the human body is small enough compared to that of the return path; however, as the communication frequency increases, effective impedance due to coupling capacitance of the return path becomes smaller and now the body impedance becomes significant so that it is no longer ignorable [2]. In addition, as the transmission distance increases, both the coupling capacitance of the external ground and the impedance of the body channel also increase, causing significant signal loss at the receiver. Hence, it is important to derive the relation between the received power, the communication frequency, the transmission distance, and the transmitting power, to fully understand the characteristics of the body channel communication; the relation can be set up by analyzing the path loss of the body channel as a function of distance, transmitting power, received power, and frequency. 


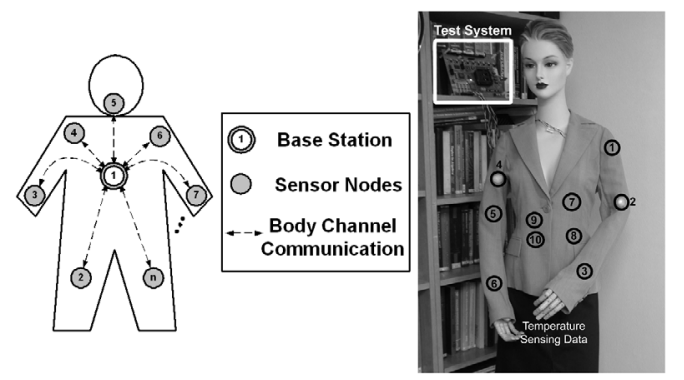

Figure 1: BCN concept (left) and the test system (right)

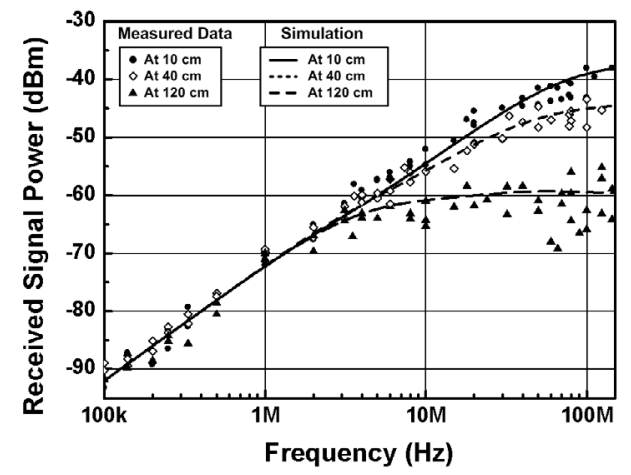

Figure 2: Measured received signal power vs. frequency

\subsection{Path Loss}

To measure the body channel path loss, we designed the transmitter boards in our previous work [2]. A circular electrode having $1.5 \mathrm{~cm}$ diameter was used to interface electrical power to the skin. Figure 2 shows the measured received signal power through the body channel with the frequency sweeping from 100 $\mathrm{kHz}$ to $120 \mathrm{MHz}$ while the transmitting power is fixed to $3 \mathrm{dBm}$ [2]. The body channel shows band-pass-like characteristics with $120 \mathrm{MHz}$ bandwidth. When the frequency is increased over 120 $\mathrm{MHz}$, the radiation effect cannot be ignored and the Federal Communications Commission (FCC) regulation limits using this band [2], [4]. From the measured data, the following relationship between the transmitting power and the received signal power is obtained [2]:

$$
P_{r}=P_{t} \cdot \frac{\alpha(K f)^{2}\left(1+f / 2 f_{0}\right)^{2}}{\left(1+f / f_{0}\right)^{p}}
$$

where $\alpha=0.0024, f_{0}=\frac{10 \times 10^{6}}{d}, p=\left\{\begin{array}{cc}4, & d>0.8 \\ 3, & \text { else }\end{array}\right.$ and $K=3 \times 10^{-9}$. Here, $P_{r}$ is the received power, $P_{t}$ is the transmitting power, $f$ is the frequency, and $d$ is the distance. From equation (1), we derive the body channel path loss, given by equation (2):

$$
\begin{gathered}
G_{d B}=10 \log _{10}\left(\frac{P_{t}}{P_{r}}\right) \\
=p \cdot 10 \log _{10}\left(1+\frac{f}{f_{0}}\right)-20 \log _{10}\left(K f\left(1+\frac{f}{2 f_{0}}\right)\right)+26.2 \\
=p \cdot 10 \log _{10}\left(1+\frac{30 d}{\lambda}\right)-20 \log _{10}\left(\frac{0.900}{\lambda}\left(1+\frac{15 d}{\lambda}\right)\right)+26.2
\end{gathered}
$$

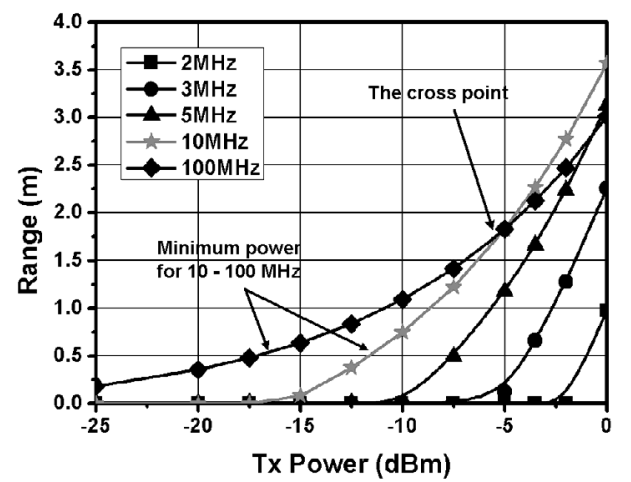

Figure 3: Maximum transmission range versus Tx power

where $\lambda$ stands for the wave length in meters.

In the following sections, we assume the received power threshold to be $-70 \mathrm{dBm}$. This is because our measurement results described in section 2 reveal that transmission range of $1.5 \mathrm{~m}$, which is a typical maximum distance of two different nodes in the body channel, results in $70 \mathrm{dBm}$ of channel loss.

\section{ANALYSIS OF BODY CHANNEL NETWORK}

\subsection{Transmitting Power}

Figure 3 shows the maximum transmission range versus transmitting power for various frequencies, plotted using equation (2). From the graph, at $2 \mathrm{MHz}$, transmitting power should be at least $-2.5 \mathrm{dBm}$ for successful transmission on the body. As the frequency increases, the minimum required transmitting power for the same transmission distance drops; it is observed that frequency band between $10 \mathrm{MHz}$ to $100 \mathrm{MHz}$ minimizes the power. At $100 \mathrm{MHz},-17.5 \mathrm{dBm}$ is enough for distance of $0.5 \mathrm{~m}$. Nonetheless, because the body channel path loss increases at frequencies over $100 \mathrm{MHz}$ as described in section 2, the cross point exists between $10 \mathrm{MHz}$ and $100 \mathrm{MHz}$ curve, around $-5 \mathrm{dBm}$ of transmitting power.

\subsection{Frequency}

Returning to equation (2), the maximum transmission range vs. frequency for various transmitting powers is analyzed. As figure 4 shows, if the transmitting power is $0 \mathrm{dBm}$, then the frequency should be higher than $1.6 \mathrm{MHz}$, or the transmitter signal cannot be

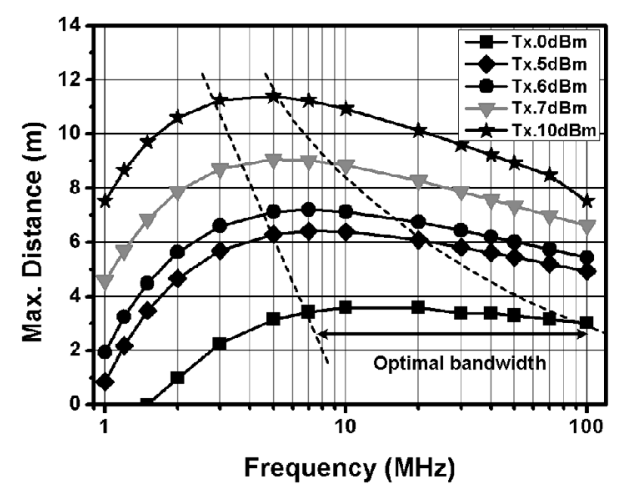

Figure 4: Maximum transmission distance versus frequency 
successfully recovered at the receiver.

In the figure, the maximum range has peak points, around 3 to $100 \mathrm{MHz}$, depending on transmitting power. Within the optimal bandwidth, the transmission range is maximized for given transmitting power. The range of over $2 \mathrm{~m}$ is not realistic considering the size of human body; however, the relation is meaningful in calculating the power-efficient frequency range. From the graph, the optimized frequency band to maximize the transmitting distance is between $10 \mathrm{MHz}$ to $100 \mathrm{MHz}$.

From equation (1), we can derive the frequency versus transmitting power for various distances, as in equation (3):

$$
P_{t}=P_{r} \cdot \frac{\left(1+\frac{f \cdot d}{10^{7}}\right)^{p}}{\alpha(K f)^{2}\left(1+\frac{f \cdot d}{2 \cdot 10^{7}}\right)^{2}}
$$

where $K, p$, and $\alpha$ are the same as given in equation (1). The equation (3) predicts that the minimum transmitting power drops sharply with frequency. This is due to the reduced channel loss in higher frequency. However, with frequencies above $100 \mathrm{MHz}$, the human body operates as an antenna, and the radiation effect begins to dominate [2].

\subsection{Topology Comparison}

Two basic topologies, a star and an ad-hoc can be used in body channel network, as shown in figure 5. Each topology is assumed to have 8 sensor (leaf) nodes placed around the body, and a base station placed in the center. In the star topology, each node is controlled by centralized coordinator, the base station, and communicates with the base station directly [6]. On the other hand, in ad-hoc topology, many identical nodes exist, and some of them will work as the message router. For simplicity, we assume the "router" nodes are positioned between the leaf nodes and the base station, as shown in figure 5. Considering the size of human body using $\mathrm{BCC}$, the transmission range is limited to $2 \mathrm{~m}$; most of the sensors will be within this range from the base station.

To perform energy simulation, the network simulator GloMoSim [5] is used. Body channel path loss model of equation (2) is imported as a channel model. Range between the sensor (leaf) nodes and the base station is varied from $0.1 \mathrm{~m}$ to $2.0 \mathrm{~m}$. Each node is set to send a constant bit stream (CBR) of 32-byte, every 0.1 to 0.4 seconds with total simulation time range to be 60 minutes. The CBR was chosen because it is most similar to the data type often used in the healthcare system, with data rate of 80 bps to $600 \mathrm{kbps}$ [1]. Transmitter power is set to $10 \mathrm{dBm}$, and the receiver threshold is $-70 \mathrm{dBm}$. Packet loss probability is limited to $0.061 \%$, and all nodes are assumed to be stationary. GloMoSim then extracts energy consumption for each node. Figure 6 shows the comparison between star and ad-hoc topologies in the view of aggregated energy consumption of all nodes. As shown in the graph, for the given distance between the base station and the sensor node, the star topology consumes about half of the energy consumed by the ad-hoc topology in the frequency region of 1 $\mathrm{MHz}$ to $110 \mathrm{MHz}$.

Typically, the ad-hoc topology has more nodes than the star topology does for the same number of the "leaf" sensor nodes. If we only consider the transmitting energy, ad-hoc topology may be the better option depending on the frequency range; however, as
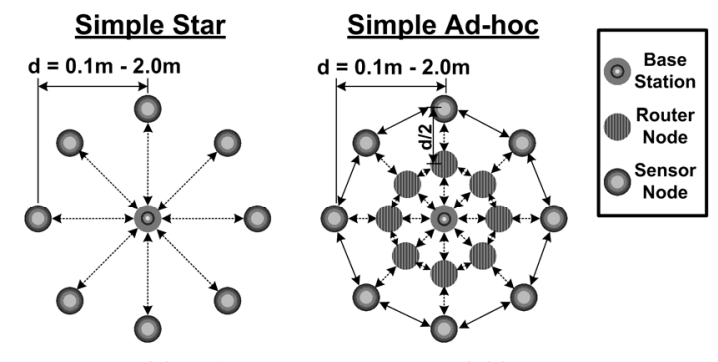

2 range $(d)>0.1 \mathrm{~m}$

Min. range $(\mathrm{d} / 2)>0.05 \mathrm{~m}$,

16 nodes +1 base station

Figure 5: Star topology vs. ad-hoc topology

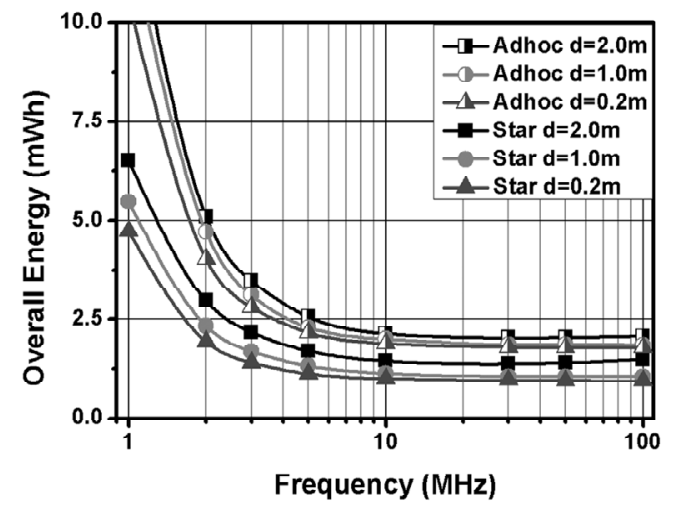

Figure 6: Star and ad-hoc energy comparison the receiver circuitry energy is roughly proportional to the number of all nodes, the overall system energy consumption is far less in star topology.

\section{PACKET DESIGN FOR BODY CHANNEL NETWORK}

\subsection{Data Packet Structure}

Figure 7 depicts the proposed physical protocol data unit of the body channel communication. The packet consists of four components: a 20-bit sync header, a 32-bit PHY header, a 16-bit footer and a variable length (0-bit to 4080-bit) payload. The sync header is split into a 4-bit preamble and a 16-bit sync ID code. The preamble field utilizes bit synchronization. The 16-bit sync ID code is reserved for scalability and for the possible future usage, e.g. in case of using other MAC layer protocols such as CDMA. The PHY header contains information on the length of the payload, and the identification for each sensor node. It is made up of a 16-bit length code, an 8-bit ID, and an 8-bit header. The length code defines the length of the payload, which varies from 0-bit to 4080-bit, with LSB 8-bit reserved. The bit width of the payload is the multiples of 16 to match the 16-bit bus in the transmitter and the receiver. The 16-bit bus is assumed because most of the biomedical signals require up to 16-bit resolution [1]. The 8-bit ID code stands for each sensor node ID, and it can

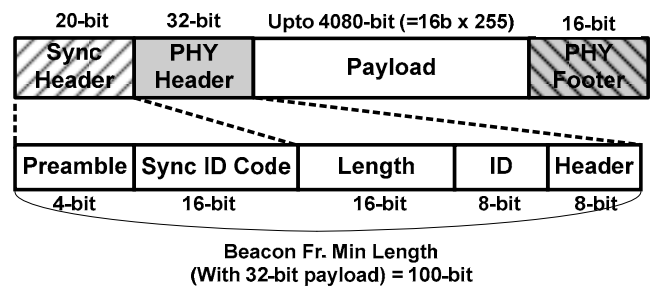

Figure 7: PHY protocol data unit structure 
distinguish up to $2^{8}=256$ different sensor nodes. The 16-bit footer is used for CRC-16 code, checking data validity of the packet.

\subsection{Energy Analysis}

With 4080-bit payload, the packet length is 4148-bit. The maximum payload size is limited to 4080 -bit so as to minimize the energy consumption. The energy consumption is analyzed for variable packet sizes by using GloMoSim [5], and the simulation environment is set similar to that of the topology comparison case, described in section 3 . The packet size is varied from 32-byte to 12,500 -byte, and the total energy consumption of the base station and the sensor node is compared for various frequencies. Because only energy is concerned, the total data size is fixed to 256kilobyte in this case; therefore, the longer the packet length, the smaller the total number of packets to be sent.

The energy consumption of every nodes for given packet size, with the total amount of data size fixed, is plotted in figure 8 . The graph shows the energy consumption drops quickly as the packet size increases, regardless of the frequency. This is because of the reduced transactions between sensor node and the base station for longer packets; each time the sensor node sends data, transmitter circuit is activated from sleep mode, which by itself consumes energy. For example, to send 256-kilobyte-data with packet size of 32-byte, the total number of packets is 8,000; for 1,280-byte, only 200 packets are required. However, as the packet size is increased over 4148-bit, the energy consumption settles around $1.02 \mathrm{mWh}, 1.20 \mathrm{mWh}$, and $1.75 \mathrm{mWh}$ within $3.4 \%, 8.3 \%$, and $19.4 \%$ deviation, respectively. In addition, if the packet is too long, then the probability of error and computational power to process the packet are increased. Therefore, the maximum packet size is designed as around 4,000-bit. Biomedical signals have various data rates ranged from $80 \mathrm{bps}$ (body temperature) to 600 kbps (EMG data), the latter having 7,500 times the formal data rate [1]. Therefore, we vary the payload length to minimize the energy consumption; we use shorter payload for ECG data than for EMG data. For example, when it comes to ECG sensors, each sensor node should send data with throughput of 15,000 bps [1]. Assuming the $10 \mathrm{Mbps}$ transmission, and assuming each sensor node is scheduled to send data every $0.25 \mathrm{~s}$, we can calculate the optimum payload size to be $16 \times 235=3760$-bit; with 4 packets, it can minimize the energy consumption.

\section{CONCLUSIONS}

The body sensor network using human body as a communication medium is analyzed in frequency, transmission range, node distance, transmitting power, received power, and energy consumption to form a power- and energy-efficient network. Analysis shows that the star topology consumes about half the energy of the ad-hoc topology for body channel network, and the optimum bandwidth for BCC is between $10 \mathrm{MHz}$ to $100 \mathrm{MHz}$. Based on the analysis, the optimum data packet structure with payload size of 0-bit to 4080-bit is designed for the body channel network.

\section{ACKNOWLEDGMENTS}

The authors acknowledge Seong-Jun Song, Sungdae Choi, Hyejung Kim, Jeabin Lee and Kyunghan Lee for their help in

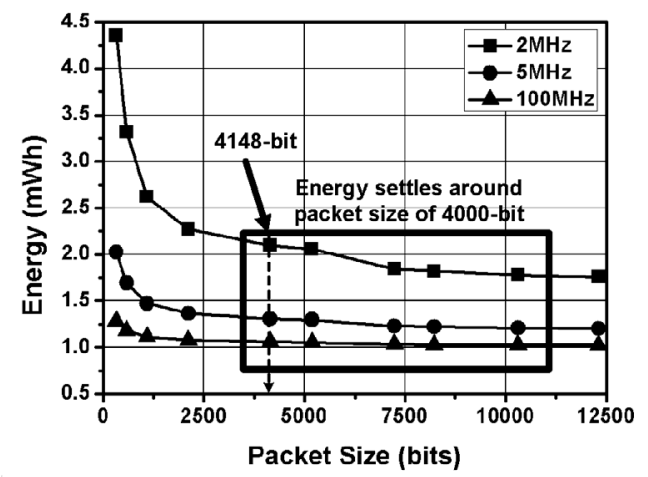

Figure 8: Energy consumption for various packet sizes when the total size of data to be sent fixed to 256-kilobyte

implementation and measurements. This work was supported by Ministry of Information and Communication (MIC) of Korea.

\section{REFERENCES}

[1] Arnon, S., Bhastekar, D., Kedar, D., and Tauber, A. 2003. A Comparative Study of Wireless Communication Network Configuration for Medical Applications. In IEEE Wireless Communications, pp.56-61. Feb. 2003.

[2] Cho, N., Yoo, J., Song, S.-J., Lee, J., Jeon, S. and Yoo, H.-J. 2007. The Human Body Characteristics as a Signal Transmission Medium for Intra Body Communication. IEEE Trans. on Microwave Theory and Techniques. vol. 55, no. 5, pp. 1080-1086.

[3] Choi, S. D., et al. 2006. A Low-power Star-topology Body Area Network Controller for Periodic Data Monitoring Around and Inside the Human Body. In Proc. International Symposium on Wearable Computers (ISWC), pp. 139-140, Oct. 2006.

[4] Federal Communications Commission, 2001. Title 47 of the Code of Federal Regulations: Part 15 - Radio Frequency Devices.

[5] GloMoSim: Global Mobile Information Systems Simulation Library. Available: http://pcl.cs.ucla.edu/projects/glomosim/

[6] Gutiérrez, J. A., Callaway, E. H., Jr., and Barrett, R. L., Jr. 2003. Low-Rate Wireless Personal Area Networks: Enabling Wireless Sensors with IEEE 802.15.4, IEEE Press, ch. 4-5.

[7] Partridge, K., Dahlquist, B., Veiseh, A., Cain, A., Foreman, A., Goldberg, J., and Borriello, G. 2001. Empirical Measurements of Intrabody Communication Performance under Varied Physical Configurations. In Proc. User Interface Software and Technology, Nov. 2001.

[8] Shinagawa, M., Fukumoto, M., Ochiai, K., and Kyuragi, H. 2004. A Near-Field-Sensing Transceiver for Intrabody Communication Based on the Electrooptic Effect. IEEE Trans. Instrumentation and Measurement, vol. 53, no. 6, pp.1533-1538.

[9] Song, S.-J., Cho, N., Kim, S., Yoo, J., and Yoo, H.-J. 2006. A 2Mb/s Wideband Pulse Transceiver with Direct-Coupled Interface for Human Body Communications. In IEEE Int. Solid-State Circuits Conf. (ISSCC) Dig. Tech. Papers, pp. 558-559. Feb. 2006.

[10] Song, S.-J., Lee, S. J., Cho, N., and Yoo, H.-J. 2006. Low Power Wearable Audio Player Using Human Body Communication. In Proc. IEEE International Symposium on Wearable Computers (ISWC), pp.125-126, Oct. 2006.

[11] Song, S.-J., Cho, N., Kim, S., Yoo, J., Choi, S. D., and Yoo, H.-J. 2007. A 0.9V 2.6mW Body-Coupled Scalable PHY Transceiver for Body Sensor Applications. In IEEE Int. Solid-State Circuits Conf. (ISSCC) Dig. Tech. Papers, pp. 366-367. Feb. 2007,

[12] Yoo, H.-J., Song, S.-J., Cho, N., and Kim, H.-J. 2007. Low Energy On-Body Communication for BSN, In Proc. International Workshop on Wearable and Implantable Body Sensor Networks (BSN 2007), pp. 15-20, Mar. 2007.

[13] Zimmerman, T. G. 1995. Personal Area Networks (PAN): Near-Field Intra- Body Communication. M.S. thesis, Media Laboratory, Massachusetts Institute of Technology (MIT). 